\title{
Polysèmes
}

Revue d'études intertextuelles et intermédiales

\section{How to Make an American Quilt de Whitney Otto: la trace et la trame}

\section{Géraldine Chouard}

\section{(2) OpenEdition}

\section{Journals}

\section{Édition électronique}

URL : http://journals.openedition.org/polysemes/1709

DOI : 10.4000/polysemes.1709

ISSN : 2496-4212

Éditeur

SAIT

\section{Édition imprimée}

Date de publication : 1 janvier 2007

Pagination : 143-164

ISSN : 0999-4203

\section{Référence électronique}

Géraldine Chouard, «How to Make an American Quilt de Whitney Otto: la trace et la trame », Polysèmes [En ligne], 8 | 2007, mis en ligne le 01 janvier 2007, consulté le 30 avril 2019. URL : http:// journals.openedition.org/polysemes/1709 ; DOI : 10.4000/polysemes.1709

Ce document a été généré automatiquement le 30 avril 2019.

Polysèmes 


\title{
How to Make an American Quilt de Whitney Otto: la trace et la trame
}

\author{
Géraldine Chouard
}

We tell ourselves stories in order to live. Joan Didion, The White Album

[T] here is no simple way to show either of our personal histories without including the other's.

How to Make an American Quilt, $160^{1}$

1 Dans How to Make an American Quilt, le patchwork se présente tout à la fois comme le thème essentiel, le principe de structuration, la figure paradigmatique par laquelle les significations se tracent et se trament, et la métaphore centrale, filée tout au long du texte.

2 Comme l'indique la formule « how to " présente dans le titre, le texte s'inscrit dans la série de ces manuels pratiques dont l'Amérique seule a le secret, abrégés de savoir-faire dont le propos est d'instruire selon le principe (normatif) qui consiste à ramener l'inconnu au connu, à partir de modèles homologués. La présence de l'adjectif "American » modifie ici les enjeux : il ne s'agit pas d'apprendre à faire un patchwork, mais un patchwork américain. De façon paradoxale, l'adjectif réduit le champ du patchwork à une catégorie particulière, mais le redéploie aussitôt à l'échelle du continent, posant par là même la question de l'identité de l'Amérique et de ses modes de représentation. Qu'est-ce qu'un patchwork américain ? Comment s'articule le lien entre Amérique et patchwork? Comment penser l'Amérique à travers ses patchworks?

3 Sous couvert didactique, la formule « How to Make an American Quilt " présente une fonction performative. Il suffit d'évoquer l'Amérique pour que surgisse la figure du patchwork, qui en est en somme l'icône. Aux plans géographique, démographique et idéologique, l'Amérique, en effet, est un patchwork ${ }^{2}$. 


\section{L'Amérique comme patchwork}

4 Tout d'abord, le lien entre l'Amérique et le patchwork s'impose visuellement: image et référent sont isomorphes. Dans Lolita, l'Amérique dans tous ses états fait figure de patchwork:

By putting the geography of the United States into motion, I did my best for hours on end to give her the impression of 'going places', of rolling on to some definite destination, to some unusual delight. I have never seen such smooth amiable roads as those that now radiated before us, across the crazy quilt of forty-eight states. ${ }^{3}$

5 On retrouve cette métaphore géographique chez Whitney Otto, où la terre américaine est nommée «God's patchwork» (15). Dans le film adapté du roman, réalisé par Patty McCormick en $1993^{4}$, la première scène est un traveling sur un quilt représentant la ville californienne de Grasse (lieu imaginaire où se situe le récit), suivi d'un fondu-enchaîné sur des vues aériennes de la région. D'une manière générale, c'est autour de la notion d'espace ${ }^{5}$ que se nouent des liens de prédilection entre Amérique et patchwork, ce dont témoignent certains noms de "quilts » exprimant un aspect de la topographie. À titre d'exemple, « Delectable Mountain » manifeste un attachement particulier à la terre :

Perhaps more than any other quilt name, the one that most confirms the love of the land, the appreciation of a vast country, the peace and plenty of opportunity was the "Delectable Mountain". The name is derived from a passage in John Bunyan's allegory, Pilgrim's Progress, published in 1678, which reads: 'They went then till they came to the Delectable Mountain... behold the gardens and orchards, the vineyards and fountains of water'. ${ }^{6}$

6 Au temps de la Conquête de l'Ouest, l'espace et le temps tendent à se confondre: le patchwork se trouve à la croisée de ces deux dimensions. Dans Mille Plateaux, Deleuze souligne "l'affinité du patchwork avec le nomadisme» et rappelle précisément sa fonction de représentation du trajet «inséparable du mouvement $»^{7}$. Les patchworks désignent parfois des parcours ou des trajectoires et font même office de journal de bord, comme en témoignent des modèles tels que «Wagon Trail », "Arkansas Traveler » ou «Wandering Foot». «Road to California» (1859) évoque ainsi, au fil d'inscriptions brodées sur les différents « blocks » $d u$ « quilt », l'histoire de la traversée du continent de deux familles :

'Left Illinois for California - April 15th, 1859', 'Crossed the plains', 'Arrived in Columbia, California on October 28, 1859', 'Seven months on the Road', 'Ho for California'. ${ }^{8}$

7 L'Amérique, en d'autres termes, s'est construite comme un patchwork, et inversement, chaque patchwork en cours de réalisation rejoue la conquête de l'Amérique. Cette transcription littérale de l'espace dans ses patchworks est peut-être à lire comme le signe de ce que Baudrillard a appellé « l'infirmité de l'Amérique vis-à-vis de l'abstraction », qui n'est elle-même que l'envers de «son génie empirique », matérialisant ainsi «sa naïveté brutale $»^{9}$, son extraversion, son sens du concret, sa liberté radicale.

8 C'est autour de cette notion d'affranchissement que s'articule encore le lien entre Amérique et patchwork. Métaphore de l'identité culturelle nationale, le patchwork libère et respecte les spécificités ethniques, sociales (contrairement au «melting pot » qui les mélange), ce qui explique, en ces temps de "political correctness», qu'elle se soit imposée. Comme le note Sacvan Bercovitch : 
The ruling elite has an American ideology; the people have their patchwork-quilt (rather than melting-pot) American multifariousness: 'America' is many forms of ethnicity, many patterns of thought, many ways of life, many cultures, many American literatures. ${ }^{10}$

9 Le patchwork se place ainsi sous le signe de la pluralité et de la diversité. C'est dans ce sens que va le discours de Jesse Jackson au congrès national démocrate de 1988 :

America is not a blanket woven from one thread, one color, one cloth. When I was a child growing up in Greenville, South Carolina, and grandmomma could not afford a blanket, [...] she took pieces of old cloth-patches - wool, silk, gaberdeen, crockersack - only patches, barely good enough to wipe off your shoes with. But they didn't stay that way very long. With sturdy hands and strong cord, she sewed them together into a quilt, a thing of beauty and power and culture. Now, Democrats, we must build such a quilt. (Democratic National Convention, Atlanta, July 1988)

De la période coloniale à l'époque contemporaine, le patchwork s'inscrit ainsi dans cette perspective multiple, gardant les traces visibles de l'hétérogénéité fondatrice dont il est «(t)issu $»^{11}$.

Depuis les années soixante, l'art du patchwork a connu un vif regain d'intérêt aux ÉtatsUnis, en particulier depuis cette exposition présentée au Whitney Museum de New York, en 1971, qui fut pour l'Amérique une occasion de se redécouvrir à travers ses patchworks. La critique littéraire féminine, et féministe, s'est depuis penchée sur ces activités textiles, perçues comme des modes d'expression méritant un examen critique spécifique. Dans un article intitulé "The Needle or the Pen ", Elaine Hedges montre comment la couture en général, et le patchwork en particulier, autrefois symboles de la domesticité féminine (associée aux notions de patience et de devoir), sont peu à peu devenus les moyens d'une créativité personnelle :

The rediscovery and celebration of women's traditional textile - the domestic arts of spinning and weaving, sewing and quilting - constitutes by now a widespread and peculiarly interesting development in contemporary feminist thinking. In the past two decades visual artists and art historians, social historians, folklorists, poets and novelists, and most recently literary critics and theorists have discovered in the processes and products of the spindle, shuttle, and needle a major source for understanding women of the past, and, as well, a source of subject matter and of images and metaphors for new creative work. ${ }^{12}$

Évoquant le patchwork comme métaphore du féminin («the pieced quilt has become one of the most central images of the new feminist art lexicon $»)^{13}$, Elaine Showalter insiste sur la valeur idéologique et politique de cette pratique, qui a servi différentes causes selon les époques. Il faut rappeler ici que les «quilting-parties» (ou "quilting-bees ») étaient pour les femmes l'occasion de quitter leur foyer et de se rencontrer, en toute légitimité, puisqu'il s'agissait de coudre ensemble et de se livrer à l'art de la récupération (le patchwork consiste en effet, avant toute chose, à faire du neuf avec du vieux). Mais c'est lors de ces «quilting-parties » que les femmes ont commencé à prendre la parole, à protester contre l'ordre établi, ou encore à militer en faveur du droit de vote. Leurs ouvrages ont souvent porté la trace de ces revendications, évoquées dans How to Make an American Quilt :

Save your opinions for your quilt. Put your heart and voice into it. Cast your ballot; express your feelings regarding industrialization, emancipation, women's suffrage, your love of family. (12)

Loin de se limiter à n'être qu'une activité intime et feutrée, le patchwork est ainsi devenu source d'émancipation féminine, stimulant l'expression de soi et l'expérimentation 
créatrice. C'est autour de cette dialectique de la contrainte et de l'autonomie, de la limite et de son dépassement que gravite pour une large part le récit de Whitney Otto.

How to Make an American Quilt s'articule autour de ces différentes dimensions (géographique, politique, idéologique) qui constituent certains des axes thématiques du texte, ce qui explique sans doute pour une part son succès (à sa sortie, le livre a connu des records de vente pendant des mois). Si le roman est dans l'air du temps, lié à des thèmes en vogue, son originalité tient à l'habileté de son agencement structurel, élaboré sur le mode du patchwork, auquel il emprunte ses principes et ses règles, et dont il décline valeurs et enjeux.

\section{Patchwork et « bri-collage »}

How to Make an American Quilt couvre un été dans la vie d'une jeune femme, Finn, étudiante en histoire, sur le point de se marier. Suivant le paradigme du déplacement géographique propre à la fiction américaine, Finn rejoint la frange de la Californie où vivent sa grand-mère et sa grand-tante, Hy Dodd et Glady Joe, pour une forme de retraite avant de connaître l'état conjugal. Conformément à la tradition qui consiste à offrir à la future mariée un "wedding quilt ", un patchwork est à l'œuvre, et le roman évoque, à travers ce "work-in-progress", l'histoire des femmes qui y contribuent. Mêlant générations, statuts sociaux, situations familiales et races, le groupe forme, pour reprendre une expression devenue presque un cliché, un tissu humain. Constitué, en l'occurrence, comme un patchwork, « an American quilt».

Le patchwork scande l'histoire de l'Amérique, et la vie des femmes. Les « quilts » étaient rituellement offerts à l'occasion de naissances ${ }^{14}$, de mariages, ou d'événements particuliers : le "Liberty Quilt ", par exemple, était un cadeau fréquemment remis aux jeunes hommes pour célébrer leur majorité. Il existe également une tradition du patchwork de deuil (« mourning quilt»), réalisé à partir des vêtements du défunt, qui est une manière de redonner corps («re-member») à ce qui avait été détruit («dismembered »). How to Make an American Quilt s'inscrit dans cette pratique traditionnelle du patchwork, qu'elle dévoie et réactualise. Un patchwork est conçu à l'intention de Finn, autour d'alliances nuptiales, reprenant le thème du classique "Double Wedding Ring ". Cependant, il n'est pas réalisé à partir de ce modèle (" pattern ») pré-établi mais cousu selon la technique de l'«appliqué », plus libre, consistant à coudre des formes prédécoupées sur l'étoffe (par opposition à la technique des "patchs", qui assemble les morceaux de tissu). Intitulé "Where Love Resides", le patchwork se définit comme «friendship quilt $»^{15}$, sur lequel chacune des participantes inscrit, sous une forme symbolique, un message personnel sur le lien amoureux. Sophia Darling coud une sirène, en souvenir de la rencontre foudroyante, à la piscine, de son futur mari, qui était tombé amoureux d'elle en la voyant plonger et l'avait abordée en disant: "When we are married, we will break tradition so you can keep your perfect name » (44). Marianna, elle, y coud un cœur, ses coups de cœur, en "crazy», symbole de sa vie sentimentale chaotique. Constance réinterprète la tradition $\mathrm{du}$ "mourning quilt », en cousant des fleurs jaunes découpées dans la chemise que portait son mari (défunt), malgré l'opposition farouche des autres quilteuses, qui trouvaient le jaune trop criard, mais conforme à l'idée que la mort devait figurer de façon discordante. Les tissus apparaissent comme métonymies de personnes ou de lieux : Anna (l'unique femme noire du groupe) coud sur un «block» un couple d'inspiration ethnique dans le tissu de la robe qu'elle 
portait le jour de la naissance de sa fille, Marianna, dont elle se souvient comme du plus beau jour de sa vie. En souvenir de son peintre de mari, qui avait fait d'elle son modèle de prédilection avant de céder aux tentations de l'infidélité, Em coud une palette de peinture dans le tissu des rideaux de son atelier, un velours vert, sombre et profond comme l'amertume, rehaussé de taches de couleurs vives comme les souvenirs de sa passion ancienne.

Il faut sans doute souligner ici l'analogie formelle entre le patchwork tel qu'il se présente dans How to Make an American Quilt, et le bricolage, tel que Lévi-Strauss le définit dans La Pensée sauvage. Art de l'incidence et de la contingence, le bricolage est une pratique empirique dont la première démarche est "rétrospective ", puisqu'elle commence par se tourner vers " un ensemble déjà constitué, formé d'outils et de matériaux ", inventorie «la totalité des moyens disponibles» pour la mise en œuvre d'un projet, engage un dialogue avec ces "objets hétéroclites" afin de "comprendre ce que chacun d'eux pourrait 'signifier' » dans un ensemble singulier, pour procéder enfin à son exécution ${ }^{16}$. Dans How to Make an American Quilt, comme en ont témoigné les exemples précédents, le patchwork se présente comme un projet collectif qui s'élabore à partir de matériaux disparates, fragments d'étoffes ayant rempli diverses fonctions antécédentes, qui se chargent d'un sens nouveau en vertu même de leur disposition dans un ensemble inédit :

Dans cette incessante reconstruction à l'aide des mêmes matériaux, ce sont toujours

d'anciennes fins qui sont appelées à jouer le rôle de moyens: les signifiés se changent en signifiants et inversement. ${ }^{17}$

17 Pour Lévi-Strauss, la réflexion mythique «apparaît comme une forme intellectuelle de bricolage » (32) en ce sens qu'elle élabore « des ensembles structurés non pas directement avec d'autres ensembles structurés, mais en utilisant des résidus et des débris d'événements ", c'est-à-dire " des bribes et des morceaux, témoins fossiles de l'histoire d'un individu ou d'une société » (32). Elle interroge ainsi événements et expériences, "qu'elle dispose et redispose inlassablement pour leur découvrir un sens » (33). How to Make an American Quilt s'inscrit dans cette perspective mythique telle que la définit LéviStrauss. Rassemblées autour de la confection d'un patchwork, procédant à coup de permutations successives, les femmes du groupe opèrent à partir de matériaux de seconde main pour produire "un objet matériel qui est en même temps un objet de connaissance » (33), agençant des éléments ou événements de leur passé respectif pour exprimer un message singulier.

18 Au plan structurel, How to Make an American Quilt se présente comme une forme de " collage » caractérisé par l'accumulation de matériaux hétéroclites : «I think of myself as a collagist, coming around and around to a point [...]. I have always written in a very broken fashion", déclarait elle-même W. Otto ${ }^{18}$. Le texte fait valoir sa diversité stylistique, juxtaposant bribes de poèmes («And each wound has the shape of your mouth", Neruda, 71), fragments de chansons populaires (Cole Porter), extraits de magazines («Beauty Tips TO MAKE HIM SAY WOW », 122), devinettes ( Q: What has four eyes and cannot see? A: Mississippi », 168), décrets de loi (sur l'esclavage), aphorismes ("Betrayal cuts both ways", 34-35) et autres pastiches ("A master is a master is a master ", 128). On note par ailleurs diverses références picturales dont les œuvres expriment, à différents titres, une certaine affinité avec le patchwork: Mondrian, Kandinsky et Miro en particulier, qui inspire Glady Joe, dont les productions s'éloignent peu à peu du figuratif : «She grows more experimental : [...] Miro-like abstracts fill vast fields of lavender, scarlet, amber» (156). Ainsi, le texte ouvre l'espace de l'énonciation 
vers la bigarrure et la disparité, niant l'unité au profit du multiple, selon une méthode que Jean-Marc Lachaud a pu qualifier de «bri-collage », visant à « éprouver le pêle-mêle des possibles $»^{19}$.

\section{«Sew to Speak » : écriture et couture}

19 Au-delà de cette esthétique du rapprochement et de la libre rencontre, le texte se construit comme un patchwork. Selon Whitney Otto, "Quilt is literally shaped like a quilt $\aleph^{20}$. Un prologue et un épilogue bordent les sept chapitres du récit, dans lesquels la narratrice prend la parole en son nom propre. À l'intérieur de ce cadre formel, la parole des autres intervenants se dispose librement. Chaque chapitre est introduit par l'image d'un «block» (motif de base répété dans le «quilt») et certains portent des titres qui pourraient être des noms de modèles de "quilts »: « Tears like Diamond Stars », «String of Pearls ", "The Crazy Quilt ». Des pointillés séparent les titres des chapitres du corps du texte et de ses différentes sections, comme autant de coutures apparentes.

Par ailleurs, l'abondance des listes qui figurent dans le texte s'inscrit dans ce principe de la juxtaposition propre au patchwork. Des listes de tissus, de couleurs, de pierres précieuses, ou de liens humains, égrenées comme des chapelets; ces mots, libérés de toute contrainte syntaxique, font valoir un droit à l'indépendance :

Take a variety of fabrics: velvet, satin, silk, cotton, muslin, linen, tweed, men's shirting; mix with a variety of notions: buttons, lace, grosgrain, or thick silk ribbon lithographed with city scenes, bits of drapery, appliqués of flora and fauna, honeymoon cottages, and clouds.

Puff them up with: down, kapok, soft cotton, foam, and stockings. (161)

Grante, schist, gneiss, rose quartz, mica, olivine, bloodstone, obsidian. (52)

Fusion, union, grafting, joining, sex, friendship, love: the difficult combination of disparate elements. (168)

21 La métaphore de la couture en littérature ne date pas de Whitney Otto. Proust disait que La Recherche avait été construite non comme une cathédrale mais «comme une robe »" Dans un registre voisin, Eudora Welty évoquait sa pratique de l'écriture en des termes proches de l'art du patchwork, puisqu'elle découpait ses textes en morceaux avant de les assembler au moyen d'épingles :

I ended up with strips - paragraphs here, a section of dialogue, and so on. I pin them together and then when I want to cut something, I cut it with the scissors. [...] You can move it, you can transpose. It's wonderful. It gives you a feeling of great moveability. ${ }^{22}$

À l'intérieur de la bordure, les chapitres de Quilt semblent également pouvoir se substituer les uns aux autres. Le récit ne suit pas une évolution chronologique, mais déambule dans le temps : les retours en arrière et les projections se télescopent tandis que les conséquences précèdent parfois les causes. D'une certaine manière, les pointillés invitent au découpage et à la recomposition : les événements pourraient ainsi s'enchaîner autrement, librement, conformément au désir de Finn, qui voulait que l'histoire ait les propriétés du vivant : «I wished for history to be vital, alive with the occasional quirk of human nature» (5). Défilent dans le désordre les différentes étapes de l'histoire américaine, de la période coloniale à la période contemporaine, en passant par la période de l'esclavage, la lutte pour les droits civiques, la guerre de Sécession ou la guerre du Vietnam. La récente manifestation "The Names Project» fait l'objet de quelques développements. Cette exposition de patchworks en plein air (un patchwork de 
patchworks, en quelque sorte), réalisés en la mémoire des personnes décédées du Sida, visait à montrer l'ampleur de l'épidémie et à témoigner de la persistance d'un lien. Conçu à San Francisco en 1988, le projet fit le tour du continent :

The newest quilt is the Names Quilt, representing those Americans who have died youthful deaths from an incurable disease. This quilt is ecclectic in its beauty [...], staggering in its implication of waste. It covers nine acres and bears nine thousand names. Say it slowly: nine thousand. [...]

The quilt weighs tons. Cloth, thread, appliqués individually weigh next to nothing but combined, bearing nine thousand patches, it is a heavy burden. [...] Be griefstricken that it represents only 20 percent of those deceased, does not even begin to measure those afflicted. (109-110)

Ce nouveau type de "quilt» a suscité des réactions diverses: s'agissait-il encore de patchwork? Certains bouts d'étoffes étaient collés, et non cousus. La tradition (celle du « mourning quilt » en l'occurrence) était-elle perdue, ou réinventée ? Pour Simon Watney (spécialiste de la question du Sida) cette nouvelle forme de patchwork prenait une nouvelle dimension métaphorique :

I was skeptical about it before I saw it. Falling back on a rationalist perspective, I thought. Oh, God, this is so sentimental. But when I finally saw it [...] it was a completely overwhelming experience. To have this social map of America. To have Liberace alongside Baby Doe, to have Michel Foucault alongside five gay cops. In many ways it's a more accurate map of America than any other I've ever seen. ${ }^{23}$

Redisposant à l'infini ses paramètres historiques et ses données sociales, le patchwork continue de définir l'Amérique, au fil de ses mutations et de ses métamorphoses.

\section{Intermezzo, rhizome, rhapsodie}

Dans Mille Plateaux, Gilles Deleuze évoque le patchwork en des termes qui offrent des perspectives de théorisation. Art de l'assemblage soumis au principe de la contiguïté, imposant un régime de fragmentation, le patchwork privilégie contact, contraste et différence, et dénonce toute unité organique (pas d'instance ordonnatrice ni de fonction de systématisation). " Avec son bout-à-bout, ses ajouts de tissu successifs infinis ", écrit Deleuze, le patchwork est «une collection amorphe de morceaux juxtaposés, dont le raccordement peut se faire d'une infinité de manières " (594-595). C'est un espace qui peut présenter des effets de "symétrie », de « résonance » (594), mais qui reste ouvert, informel, non hiérarchisé. Pour penser cet espace, Deleuze introduit la métaphore du rhizome (mouvant, multiple, différencié, non génératif) par opposition à la métaphore de l'arbre (centré, ramifié, hiérarchique) :

Ce qui est mis en question dans le rhizome, c'est un rapport [...] tout différent du rapport arborescent : toutes sortes de 'devenirs'. [...]

Un rhizome ne commence pas et n'aboutit pas, il est toujours au milieu, entre les choses, inter-être, intermezzo. L'arbre est filiation, mais le rhizome est alliance, uniquement alliance. L'arbre impose le verbe 'être', mais le rhizome a pour tissu la conjonction 'et... et... et...' Il y a dans cette conjonction assez de force pour secouer et déraciner le verbe être. ${ }^{24}$

Le patchwork présente cette structure rhizomatique, qui crée de nouvelles alliances à partir d'un matériau « déjà-là ». C'est un art du recyclage qui fait surgir le sens par le biais de la juxtaposition de pièces constitutives («odds and ends», 177) selon des axes qui peuvent changer : un patchwork offre toujours plusieurs types de lectures, qui varient elles-mêmes avec le temps. À titre d'exemple, les nœuds papillon du modèle du même 
nom, « Bow Tie », peuvent faire figure de bobines de fils, selon la texture, l'intensité et le mode d'agencement des tissus employés. Finn projette ainsi l'évolution, avec le temps, des figures du patchwork en cours de réalisation: «perhaps some emerging images will be lambs or yellow roses or mermaids, entwined wedding rings or hearts in states of disrepair » (8).

Dans How to Make an American Quilt, le patchwork se définit par son caractère informel, privilégiant l'échange entre des êtres réunis du seul fait de leur choix, par affinité, partageant au fil de leur production une part de leur histoire. Dans ce groupe, «les initiatives locales sont coordonnées indépendamment d'une instance centrale ${ }^{25}$ : Anna Neale, la fondatrice du groupe ne joue pas de rôle d'encadrement, mais laisse chacune élaborer son projet selon son désir. Un réseau de liens et d'alliances se trame alors dans cet espace placé sous le signe de la simple conjonction.

Rhizomatique, le patchwork manifeste également une dimension rhapsodique. Au sens premier du terme, puisque rhapsodie vient de rhaptein, du grec "coudre", mais également au sens musical du terme, car le patchwork est affaire d'affinité et d'harmonie. La toute première consigne de la narratrice va dans ce sens :

When you choose your colors, make them sympathetic to one another. [...] Think of music as you orchestrate the shades and patterns; pretend that you are a conductor in a lush symphony hall. (8)

La «quilting-party" semble elle-même faire l'objet d'une chorégraphie particulière, silencieuse et tacite :

Sometimes you can tell what is on their minds from what they avoid saying or the way in which they say it. Or their seating arrangement for the evening. You would think that it would always be the same, unchanged, but it is not. I am reminded of some sort of complicated, intricate dance of many partners, facing many different directions. (176)

Alors qu'elle observe le patchwork réalisé pour elle, Finn écoute ce qui se dit ouvertement et ce qui s'exprime en filigrane, plus souterrainement, lorsque la rhapsodie se fait rhizome :

You will find this work to be most revealing, not only in the material contributions to the quilt, but in who enjoys sewing them and who does not. This random piecing together. (8)

Le patchwork est affaire d'affect et de voix. Il n'est que la somme de ce qu'on y met, comme l'avait annoncé la devise du prologue : " put your heart and voice into it » (12). Pour Anna Neale («the founder of the Grasse Quilting Circle »), l'aiguille a été le premier outil d'expression de $\operatorname{soi}^{26}$. Ce qui redonne à l'expression « sew to speak» son sens plein :

I am not invisible because of this closed circle. I am not unknown. I learned to speak with needle and thread long before society finally 'gave' me a voice - as if society can give anyone a voice; it can only take a voice away. (133)

How to Make an American Quilt consacre quelques-unes de ses pages à des rappels historiques relatifs aux patchworks des esclaves, et notamment à "The Life Before", cousu par l'arrière-arrière grand-mère d'Anna, qui avait évoqué à travers ce « quilt » en appliqué l'histoire de ses ancêtres :

Divided into fifteen large squares filled with appliquéd animals, birds, men of dark brown, hovering angles, serpents as large as life, stars, the outsized sun, flaming candles of dripping wax. These were African scenes: animals with tusks, warriors clashing with spirits and themselves and beasts. (134) 

be » (89), dit Constance à Marianna. «Free love and no ties that bind », telle est la devise de Sally (159). Ce principe de juxtaposition a toutefois ses limites et ses dangers, basculant vers l'indifférence ou la désobéissance ${ }^{30}$. Glady Joe et Arthur auront ainsi passé leur vie l'un auprès de l'autre, sans jamais pouvoir se rejoindre : «trying and failing at physical intimacy, never quite making contact » (18). À l'inverse, la juxtaposition peut devenir licence, jusqu'à la transgression : plusieurs sont évoquées, la plus frappante étant une liaison entre Hy et Arthur, le jour de l'enterrement du mari de Hy, décrite comme « a onetime thing " (30). Cette trahison (les deux femmes sont sœurs) donne lieu à une colère spectaculaire lors de laquelle Glady Joe casse la vaisselle de la maison jusqu'à la dernière assiette. Une fois la fureur passée, elle récupère tous les morceaux brisés pour en faire 
une immense mosaïque murale. To re-member, c'est-à-dire pour ne pas oublier et tenter tout à la fois de se reconstruire :

Since she began this project, a week after the throwing frenzy, she has felt a sense of purpose and calm, as if this is the only way she can somehow go on with her life - transforming these pieces of junk, swept up from her bedroom and bathroom floors, into art. [...] She did not know what to expect when she began the project, she only knew that it helped to hold her fractured life together. (33)

Alors que son mari lui propose de tout jeter, Glady Joe pense intérieurement :

Arthur, I am not the kind of person who throws away something because it is broken. I would not waste what could still be of use. (32)

Tout au long du récit, le patchwork porte les traces de tels déplacements d'affects. Une fois réconciliées, les deux sœurs se remettent à coudre ensemble, mais projettent dans le patchwork différends et différences. Glady Joe ne veut plus représenter de personnages, seulement des paysages et des monuments :

[Hy] is encouraging Glady Joe to agree to do Monticelli but Glady Joe will only agree if they leave out the remaining slave quarters in the design. [...] (There are only two ways to deal with tragedy and injustice: show it plainly or hide all traces; and the sisters are in disagreement on this matter.) (15)

$\mathrm{Hy}$ is responsible for something else, something that Glady Joe cannot entirely forgive. Glady Joe prefers stitching landscapes and monuments. People can hurt you, betray you. People are dangerous if you are not careful. (16)

Le patchwork permet de s'exprimer - «to shout out loud in silk and bits of old scarves » - , de se faire entendre - « [to] make yourself heard in a wild profusion of colors, shapes, themes, and dreams with your fingertips » (128) - sans avoir à s'expliquer :

Remember, you do not need to tell anyone what your contributions mean and it is more likely they will hold meaning for you alone anyway. Do not explain. This is your right. (179)

Pudique, le patchwork présente aussi une forme d'indécence, dans la mesure où il porte la trace d'affects personnels, dévoilant toujours ainsi une part de soi. Composé à partir de tissus chargés d'une histoire, il transmue un temps diachronique en une totalité synchronique close sur elle-même. C'est un «lieu de mémoire » qui fait figurer la profondeur à la surface. Comme en témoigne cette "quilteuse » anomyme du siècle dernier, le patchwork rassemble et reflète les divers épisodes d'une existence singulière :

It took me more than twenty years, nearly twenty-five, I reckon, in the evenings after supper when the children were all put to bed. My whole life is in that quilt. It scares me sometimes when I look at it. All my joys and my sorrows are stitched into those little pieces. When I was proud of the boys and when I was downright provoked and angry with them. When the girls annoyed me or when they gave me a warm feeling around my heart.

And John too. He was stitched into that quilt and all the thirty years we were married. Sometimes I loved him and sometimes I sat there hating him as I pieced the patches together. So they are all in that quilt, my hopes and my fears, my joys and my sorrows, my loves and hates. I tremble sometimes when I remember what that quilt knows about me. ${ }^{31}$

41 Le patchwork devient en quelque sorte l'album d'une vie. C'est d'ailleurs le titre de certains modèles ( Album Quilts »), qui apparaissent comme les premiers textes féminins de la nation américaine. Une manière de se dire, de donner de l'étoffe à ses expériences et à ses pensées. Et de remettre de l'ordre dans le disparate. Sophia Darling fait allusion à cette fonction apaisante et unificatrice du patchwork, qui refonde un régime continu à partir d'éléments disjoints sans effacer la trace des raccords et des jointures : 
Sophia Darling wants to tell Preston that she loves him, wants to be less rule bound with her children; but instead she spends one night a week piecing together bits of fabric with a group of women. As if she could piece together all the things she feels inside, stitch them together and make everything seem whole and right. (58)

Il faut ainsi voir dans le patchwork comme un espace qui trouve tout à la fois son origine et son mode de fonctionnement dans cette dynamique de la dislocation et de la réunification, de la mise en pièce et de la (re)mise en forme.

\section{"A Stitch in Time Saves Nine » : patchwork et éducation sentimentale}

42 Suivant le principe qu'un patchwork en cache toujours (au moins) un autre, selon les effets d'optique qu'il est susceptible de produire, How to Make an American Quilt se définit par son ambivalence générique : manuel de patchwork, le texte est aussi une éducation sentimentale, qui pourrait s'intituler «What to Expect from Marriage ». Les deux registres se rejoignent et s'entrecroisent pour épaissir la trame narrative d'un texte qui finit par se résorber dans la continuité d'un seul tissu.

Fidèle aux promesses annoncées dans le titre, How to Make an American Quilt fournit de très nombreux éléments d'information d'ordre historique et technique, richement documentés :

You need a large wooden frame and enough space to accommodate it. Put comfortable chairs around it, allowing for eight women of varying ages, weight, coloring, and cultural orientation. (7)

Your needles must be finely honed so you don't break the weave of your fabric. The ones from England are preferable. And plenty of good quality thread, both to bind the pieces and adorn the quilt. (8)

The Crazy Quilt was a fad of the nineteenth century and as such is not truly considered Art, yet still it has its devotees. It is comprised of remnants of material in numerous textures, colors; actually, you could not call the squares of a Crazy Quilt squares, since the stitched-together pieces are of all sizes and shapes. This is the pattern with the least amount of discipline and the greatest measure of emotion. (8) Quilts are comprised of spare time as well as excess material. (32)

Mais à mesure que se déroule le fil de l'histoire, ces développements thématiques s'articulent à d'autres propos d'ordre privé. Le "you » impersonnel des consignes du manuel dérive vers le particulier, jusqu'à l'intime :

Take some material from clothing that belongs to some family member or friend or lover (if you find yourself to be that sort of girl). Bind them together carefully. Wonder at the disparity of your life. (9)

Hold your secret regarding this quilt. What upsets you.

What puts you out. Your inner conflict regarding the vagaries of control. (39)

Sophia prefers the true challenge of a traditional, established pattern. That is the true challenge she thinks - to work within a narrow confine. To accept what you cannot have; that from which you cannot deviate. (61)

Buy a large, 'quilt-size' piece of Plexiglas. Leave the back open for the work to 'breathe'. Nothing, you know, can be kept inviolate, in a box, away from the harm that can be inflicted on a marriage by a wandering husband who has often told you that he cannot help but wander. (93)

C'est en ce sens que l'on peut qualifier ce texte de sentimental, au sens le plus fort du terme. À travers ces consignes, le patchwork offre ainsi une longue méditation sur l'art du lien, qu'il s'agisse du lien conjugal, des liens du sang, des liens d'amitié, ou encore des 
liens sociaux. Entre attaches et astreintes, symbioses et déchirures, liaisons et décalages, How to Make an American Quilt décline l'extrême variété des situations des êtres mis en relation. Le passage du registre de la couture à celui du lien s'opère au moyen de fondusenchaînés qui procèdent dans un sens, ou dans l'autre :

Meditate on the soul-kiss, which, prior to the twentieth century, meant that the souls of the lovers were exchanged; transferred from one to the other as a great gift and act of faith. But the body grows lonely for its own soul (even as it loves the new one) and longs to have it returned. A quilt, though stitched together, will always be separate, individual parts. (68)

C'est ainsi que les formules s'éclairent mutuellement, se confèrent une intelligence réciproque, de sorte qu'il arrive parfois que l'on ne sache plus ce qu'on lit. La formule «Watch for breakage » (68), par exemple, trouve sa force dans l'ambivalence du message qu'elle délivre ${ }^{32}$. D'une manière générale, l'une des fonctions essentielles du patchwork semble être de faire valoir la vulnérabilité fondamentale de l'existence humaine placée sous le signe du lien :

And remember, no matter how careful you are, you might not be able to prevent some damage to your quilt - no matter how much you are to it, how much of your skill and time you have invested in it or how carefully you followed all the rules for care, something unforeseen may ruin it beyond repair, leaving only the memory of the quilt behind. Do not castigate yourself; you may not be to blame. You did your best. These are fragile textiles. These things happen. (109)

47 Le processus de déplacement à l'œuvre dans le patchwork fonctionne également au plan linguistique : le texte est ainsi parcouru d'un certain nombre de métaphores empruntées au registre de la couture. L'image de l'ourlet resurgit dans l'expression "hemmed in " pour traduire le sentiment d'oppression de Constance face aux exigences incessantes de son mari :

There were days when she felt hemmed in, crowded in her own house, as if Howell were not one person but a small group that made continuous, inconsequential demands on her time and patience, which, taken together, resulted in an enormous demand that she could never satisfy. (81) (C'est moi qui souligne)

48 À l'inverse, l'adverbe "seamlessly $»^{33}$ exprime une passion (romanesque) sans mélange, qui plonge Finn dans la méditation:

When Heathcliff ran away from Wuthering Heights, he left Cathy wild and sad, howling on the moors, I am Heathcliff, as if their love was so powerful, their souls so seamlessly mated, that no division existed for them, save the corporeal (though I tend to believe they got 'together' at least once), which is of little consequence in the presence of the spirit.

All of which leaves me wondering, astonished, and a little put off. How does one accomplish such a fusion of selves? And if the affection is that strong, how does one avoid it, leaving a little room for the person you once were? (5)

Cette tension entre contrainte et fusion reformule la dialectique propre au patchwork qui déracine et reconstruit, prélève et rassemble : « The impulse to unify and separate, rend and join, is powerful and constant » (176), exprimant à chaque recomposition une vision unique. Évoquant "la part interventionniste qui préside à la création de toute œuvre ", Bertrand Rougé souligne la "volonté d'unir et d'unifier " qui la caractérise, "mais d'abord un immense désir d'altération, un besoin intense et presque furieux, c'est-à-dire fou, de séparer, de trancher, de faire le geste à deux mains de fendre et écarter ». De sorte que « d'un même mouvement, de nouveaux objets sont créés, une place leur est assignée et un nouvel ordre nait qui est un nouveau monde $»^{34}$. How to Make an American Quilt 
donne à cette médiation sa pleine dimension : la question en effet est moins de savoir comment faire un patchwork américain que de le faire, d'en faire un. Comme l'écrit Michel Cusin :

On ne peut jamais tout dire, ni être tout à tous. On ne peut dire que quelque chose à quelqu'un, quelque part..$^{35}$

Sous ses airs syncrétiques et rassembleurs, le patchwork est un art du particulier, relevant du "quelque chose » "quelque part », œuvre de "quelqu'un », ou de quelquesuns. Une affaire souvent collective mais toujours singulière: si d'une certaine manière, c'est toujours la même histoire (de fil, de tissu, de composition), précisément ce n'est jamais la même. Cousu au fil du temps, autour duquel il se trame, et dont il porte la trace, le patchwork se réinvente dans chacun de ses "quilts", se construit autour du désir «d'altération » qui en fait la force: «Only you can tell your story» (137), disait Anna, avant d'ajouter, en fin de parcours: "There is no simple way to show either of our personal histories without including the other's » (160). Le fil du patchwork toujours mène à l'autre. Tâche immémoriale, infinie, infiniment recommencée, le patchwork, comme le lien à l'Autre, comme l'Amérique sans doute, reste à faire, à construire, à découvrir. Pour dire et redire quelque chose, quelque part, à quelqu'un.

\section{NOTES}

1. Whitney Otto, How to Make an American Quilt, New York: Ballantine Books, 1991. Les références suivantes renvoient à cette édition.

2. En anglais, le mot "patchwork » désigne le mode d'assemblage des pièces entre elles, « quilt » le produit fini, et «quilting » la technique de matelassage. En français, « quilt » se traduit le plus souvent par «patchwork». Pour un lexique complet sur le patchwork, voir Michèle Dhont \& Suzon de Marcilly, Patchwork. Histoire et technique, Paris : L'Inédite, 1991, 187-189.

3. Vladimir Nabokov, Lolita (1955), Harmondsworth: Penguin, 1984, 152. C'est moi qui souligne. Métaphore géographique, le patchwork (désigné plus loin comme «crazy quilt of dark and pale fields », 306), présente une valeur idéologique : «crazy » renvoie à l'absence de hiérarchie qui préside à son mode de structuration, ici jusqu'à la transgression, au sens où Humbert fait croire à Lolita qu'ils sont en train de découvrir l'Amérique, alors qu'en réalité il se délecte, au fil des kilomètres, de cet espace pluriel, placé sous le signe de la simple juxtaposition (Humbert et Lolita traversent l'Amérique côte à côte mais ne feront jamais la route ensemble).

4. Le film, How to Make an American Quilt, est sorti en France en 1996 sous le titre Le Jour du patchwork. La réalisation du film a fait l'objet d'un livre, très documenté. Cf. Patty McCormick, Pieces of an American Quilt: Quilts, Patterns, Photos, \& Behind the Scenes Stories from the Movie, Lafayette, California: C\&T Publishing, 1996. Il a été traduit en français sous le titre Quilts américains et publié aux Éditions DMC, dans la collection « Le Temps apprivoisé » en 1997.

5. Et au-delà, tout ce qui est «Americana » (cartes, drapeaux, bannières, aigles, statue de la liberté) constituent les motifs privilégiés du patchwork américain.

6. Patsy and Myron Orlofsky, Quilts in America, New York: Abbeville, 1992, 281.

7. Gilles Deleuze et Félix Guattari, Mille Plateaux, Paris : Éditions de Minuit, 1980, 595- 596. 
8. Cité par Elaine Hedges, "Quilts and the Westward Movement ", in Hearts and Hands: Women, Quilts and American Society, Nashville: Rutledge Hill Press, 1987, 56.

9. Jean Baudrillard, Amérique, Paris : Grasset, 1986, 93 \& 31.

10. Sacvan Bercovitch, Ideology and Classic American Literature, cité par Elaine Showalter, Sister's Choice: Tradition and Change in American Women's Writing, Oxford: Oxford UP, 1994, 168.

11. J'emprunte la formule à Bertrand Rougé, «Le collage ou le coup de force ", in Collages/ Montages, actes du second colloque du CICADA, décembre 1991, Pau : PU de Pau, 1993, 9.

12. Elaine Hedges, " The Needle or the Pen: The Literary Discovery of Women's Textile Work », in Florence Howe (ed.), Traditions and the Talents of Women, Chicago: U of Illinois P, 1991,338.

13. Elaine Showalter, Sister's Choice, op. cit., 161.

14. Lorsqu'elle était enceinte de sa fille Marianna, Anna avait cousu pour elle un «quilt» pour son berceau (" a crib quilt») de type Amish, sur le modèle «Broken Star », préfigurant ainsi sa destinée tout à la fois lumineuse (idolâtrée par sa mère) et brisée (Marianna ne connaîtra jamais son père).

15. Dans le film, le patchwork est réalisé à partir d'un modèle ancien, «Baltimore Album Quilt », datant de 1873. Il semble qu'il s'agisse du modèle représenté dans Sister's Choice, op. cit., 152, fig. 2.

16. Claude Lévi-Strauss, La Pensée sauvage, Paris : Plon, 1962, 26-32.

17. Ibid., 31.

18. Roxane Farmanfarmain, "Whitney Otto: A Transcendent Passion », Publishers Weekly Interview, 2 June 1997, 45.

19. «Des pratiques collagistes, ou de la nécessité d'éprouver le pêle-mêle des possibles », in Montages/Collages, op. cit., 105-113.

20. Roxane Farmanfarmain, op. cit., 45.

21. Marcel Proust, Le Temps retrouvé, Paris : Gallimard, coll. «Bibliothèque de la Pléiade », 2, III, 1033-1034.

22. Peggy Whitman Prenshaw (ed.), Conversations with Eudora Welty (1984), New York : Washington Press, 1985, 272-273.

23. Cité par John Seabrook, «The AIDS Philosopher », Vanity Fair, Dec. 1990, 111.

24. Gilles Deleuze et Felix Guattari, Mille Plateaux, op. cit., 32 \& 36.

25. Ibid., 21, note 14 .

26. Dans "The Needle or the Pen», Elaine Hedges retrace cette évolution des moyens d'expression féminine au fil du temps (Traditions and the Talents of Women, op. cit., 338-364).

27. Elaine Hedges, Hearts and Hands, op. cit., 48.

28. "a percussive manner of handling textile color", "an exuberant acceptance of contingency ", telles sont, selon Robert Ferris Thompson, les caractéristiques propres à ce type de «quilt». Cf. " From the First to the Final Thunder: African-American Quilts, Monuments of Cultural Assertion ", in Eli Leon, Who'd A Thought It: Improvisation in African-American Quiltmaking, San Francisco Craft and Folk Art Museum, 17 \& 21.

29. Cité par Elaine Hedges, Hearts and Hands, op. cit., 15 \& 70.

30. C'est précisément le titre d'un ouvrage de Claude Fauque et Marie-Noëlle Fayard, Le Patchwork ou la désobéissance, qui met en évidence la pleine liberté créatrice liée à cet art qui se réinvente à chaque patchwork (Paris : Dans le droit fil, 1993).

31. Anonymous $19^{\text {th }}$ century quiltmaker, cité par Marguerite Ickis, The Standard Book of Quiltmaking and Collecting, New York: Dover, 1949, 270. C'est moi qui souligne.

32. C'est sans doute ce chatoiement du sens qui a manqué au film, globalement plus littéral, plus plat.

33. On retrouve cet adjectif plus loin, pour évoquer la fusion génétique dont résulte un être humain: "But the aggregate that is you is so seamless, so smooth, that no one - including yourself - can see where your father's lines leave off and your mother's pick up » (162).

34. Bertrand Rougé, « Le collage ou le coup de force », op. cit., 9. 
35. Michel Cusin développe ce passage autour de la notion de "Pas-Tout " chère à Lacan, articulée sur le principe de l'impossibilité à dire sans défaire, disjoindre, exclure. Cf. «Parole et symptôme dans la parabole », in Jean Delorme (éd.), Parole-Figure-Parabole, Lyon: PU de Lyon, 1987, 47.

INDEX

oeuvrecitee How to Make an American Quilt 